\title{
Condensation of a One-Dimensional Lattice Gas
}

\author{
Kurt Johansson * \\ Department of Mathematics, Royal Institute of Technology, Stockholm, Sweden ${ }^{\star \star}$
}

Received February 6, 1990; in revised form April 16, 1991

\begin{abstract}
We consider a one-dimensional lattice gas in the canonical ensemble with interaction energy $1 / r^{\alpha}, 1<\alpha \leq 2$. Using an energy-entropy argument we show that the gas condenses at sufficiently low temperatures meaning that the gas has a non-uniform density in the thermodynamic limit.
\end{abstract}

\section{Introduction}

Consider a one-dimensional lattice gas in the canonical ensemble, i.e. the number of positions in $[1, L] \cap Z$ occupied by particles is a fixed number $N$. Let the interaction energy between two particles at distance $r$ be $-1 / r^{\alpha}, 1<\alpha \leq 2$. We will show that, with appropriate boundary conditions, this lattice gas condenses at sufficiently low temperatures, meaning that the gas has a non-uniform density in the thermodynamic limit $N, L \rightarrow \infty, N / L \rightarrow \varrho, 0<\varrho<1$.

The proof is an energy-entropy argument which makes rigorous the following heuristic argument, see Landau-Lifshitz [6] abd Thouless [9]. Consider configurations that are not condensed already, say those that are approximately uniform. Suppose that we can partition these configurations into blocks $A_{1}, B_{1}, A_{2}, \ldots, A_{k}, B_{k}$, where almost every position in $A_{1}, \ldots, A_{k}$ is occupied and almost every position in $B_{1}, \ldots, B_{k}$ is empty. Also assume that all these blocks have length $\geq d$. Rearrange the blocks so that the condensed configuration $A_{1} A_{2} \ldots A_{k} B_{1} \ldots B_{k}$ is obtained. The change in energy is $\Delta E \sim-C k E_{\alpha}(d)$, where $E_{\alpha}(d)=d^{2-\alpha}$ if $1<\alpha<2,=\log d$ if $\alpha=2$ and $\leq$ const. if $\alpha>2$. The change in entropy due to many configurations being mapped to the same condensed configuration is roughly $\Delta S \sim-\log \left(\begin{array}{l}L \\ k\end{array}\right) \sim-k \log (L / k)$. The free energy thus changes by $\Delta F=\Delta E-\beta^{-1} \Delta S \sim k\left(-C E_{\alpha}(d)+\beta^{-1} \log (L / k)\right)$. If $\alpha>2$ and $k=o(N)$,

\footnotetext{
* Research supported by the Swedish research councils NFR and STUF

$\star \star$ Mail address: Department of Mathematics, University of Uppsala, S-752 38 Uppsala, Sweden
} 
then we cannot have $\Delta F<0$ with $\beta$ finite and $N \rightarrow \infty$. If $\alpha=2$ then $\Delta F<0$ if $d \geq(L / k)^{1 / \beta C}$, and thus in this case the system could condense. Similarly for $\alpha<2$ with a smaller $d$. This explains why we can expect a condensation when $\alpha \leq 2$ but not when $a>2$. We see that in order for the above argument to work $d$ must be sufficiently large. If we do rearrangements only locally a smaller $d$ is possible. In the proof we will partition every configuration into blocks which are alternatingly dense and dilute. By making local rearrangements we obtain configurations with sufficiently long blocks to make the global rearrangement sketched above work.

The existence of a phase transition in a one-dimensional system with longrange interaction was first established by Dyson [2], who showed that there is spontaneous magnetization in the $1 d$ Ising model with interaction $J_{i j} \sim 1 /|i-j|^{\alpha}$, $1<\alpha<2$, at sufficiently low temperatures. That this also holds for $\alpha=2$ was shown by Fröhlich and Spencer [4]. Recently the understanding of the case $\alpha=2$ has improved considerably by use of the relation between the Ising model and certain percolation models. In particular the existence of the Thouless effect was proven. See Aizenman, Chayes and Newman [1]. Thus the existence of a phase transition in long-range $1 d$ lattice models is not a new result. The novelty in this paper lies in the character of the proof, where we work in the canonical ensemble and establish the phase transition as a condensation. In an accompanying paper, Johansson [5], we will show how the same ideas can be extended to prove the existence of a liquid-gas transition in a one-dimensional continuous model.

The organization of the paper is as follows. In Sect. 1 we define the model and state our results. In Sect. 2 we define the partition of a configuration into blocks and the local rearrangement procedure. We then, in Sect. 3, state lemmas giving the change in energy and entropy due to the local rearrangement. These two lemmas are combined into an energy-entropy argument for the local rearrangement and we can then perform the global rearrangement and prove the main theorem. The proof of the entropy and energy lemmas occupy Sects. 4 and 5. The last section contains an argument which proves that, if the free energy is a strictly convex function of the density, then no condensation occurs. As shown in the first section this implies that at high temperatures the gas does not condense.

\section{Definitions and Results}

\subsection{Definition of the Model}

Let $\Lambda=[1, L] \cap Z, \Lambda^{c}=Z \backslash \Lambda$, where $L$ is a positive integer. With each site $i \in \Lambda$ we associate a random variable $n_{i}$ taking the values 0 and 1 and satisfying the constraint

$$
\sum_{i \in \Lambda} n_{i}=N
$$

where $N$ is a positive integer. If $n_{i}=1$ we say that the site $i$ is occupied by a particle, otherwise it is empty. Equation (1.1) thus says that the total number of particles is fixed. As boundary conditions we set $n_{i}=1$, if $i \in Z \cap(-\infty, 0]$ and $n_{i}=0$ if $i \in Z \cap[L+1, \infty)$. We let $\underline{n}=\left(n_{i}\right)_{i \in Z}$ and call this a configuration in $\Lambda$ with the specified boundary conditions. $Q=Q_{N, L}$ denotes the set of all 
configurations in $\Lambda$. The Hamiltonian is

$$
H(\underline{n})=H_{N, L}(\underline{n})=-\sum_{\substack{i \in A, j \in Z \\ i \neq j}} \frac{n_{i} n_{j}}{|i-j|^{\alpha}},
$$

where $\alpha>1$. We will work with the canonical ensemble probability measure which is defined by

$$
P(A)=P_{\beta, N, L}(A)=Z^{-1} \sum_{\underline{n} \in A} \exp (-\beta H(\underline{n})),
$$

where $A \subseteq Q, \beta$ is the inverse temperature and

$$
Z=Z(N, L)=\sum_{\underline{n} \in Q} \exp (-\beta H(\underline{n}))
$$

is the partition function.

For $x, y \in Z, x \leq y$ and a given configuration $\underline{n}$ we define

$$
N(x, y)(\underline{n})=\sum_{i: x \leq i \leq y} n_{i}
$$

$N(x, y)(\underline{n})$ is the number of particles in $[x, y]$.

Let $\varrho, 0<\varrho<1$, denote the asymptotic average density, and let $A \rightarrow Z^{+}$ denote the thermodynamic limit $N, L \rightarrow \infty, N / L \rightarrow \varrho$. For $0<\tau_{1}<\tau_{2}<1$ and $\underline{n} \in Q$ we define

$$
d\left(\tau_{1}, \tau_{2}\right)(\underline{n})=\frac{1}{L\left(\tau_{2}-\tau_{1}\right)} N\left(\left[\tau_{1} L\right],\left[\tau_{2} L\right]\right)(\underline{n}),
$$

where [.] denotes the integer part.

Definition 1.1. We say that the gas has uniform density in the thermodynamic limit if for each $\varepsilon>0$ and any fixed $0<\tau_{1}<\tau_{2}<1$,

$$
\lim _{\Lambda \rightarrow Z^{+}} P\left\{\underline{n} \in Q ; \varrho-\varepsilon \leq d\left(\tau_{1}, \tau_{2}\right)(\underline{n}) \leq \varrho+\varepsilon\right\}=1 .
$$

Otherwise we will say that the gas has non-uniform density in the thermodynamic limit.

\subsection{Results}

For a given small $\delta>0$ and density $\varrho, 0<\varrho<1$, we put

$$
d_{1}=(1-\delta)^{-1}(\varrho-\delta), \quad d_{2}=(1-\delta)^{-1} \varrho .
$$

The main result of this paper is the following theorem.

Theorem 1.2. Let $1<\alpha \leq 2$. There exist positive constants $K$ and $\kappa$, depending only on $\alpha$, and $\beta_{0}=\beta_{0}(\varrho, \alpha)$ such that if $\beta \geq \beta_{0}$ and $\delta=K \exp (-\kappa \beta)$, then for each $\varepsilon>0$,

$$
\lim _{\Lambda \rightarrow Z^{+}} P\left(\underline{n} \in Q ; d\left(\tau_{1}, \tau_{2}\right)(\underline{n}) \geq 1-2 \delta\right)=1
$$

for any fixed $0 \leq \tau_{1}<\tau_{2} \leq d_{1}-\varepsilon$, and

$$
\lim _{\Lambda \rightarrow Z^{+}} P\left(\underline{n} \in Q ; d\left(\tau_{1}, \tau_{2}\right)(\underline{n}) \leq 2 \delta\right)=1
$$

for any fixed $d_{2}+\varepsilon \leq \tau_{1}<\tau_{2} \leq 1$. 
Thus for sufficiently high $\beta$ the gas has non-uniform density in the thermodynamic limit. We also want to show that for sufficiently small $\beta$ we have uniform density in the thermodynamic limit. To do this we will use the free energy of the lattice gas. The free energy, $f(\varrho, \beta)$, is defined by

$$
\beta f(\varrho, \beta)=-\lim _{\Lambda \rightarrow Z^{+}} \frac{1}{L} \log Z(N, L),
$$

where $Z(N, L)$ is the partition function defined above. It can be shown that this limit exists if $\alpha>1$, is a convex function of $\varrho$ and does not depend on the choice of boundary conditions, see Ruelle [8]. In Sect. 6 we will prove the following theorem.

Theorem 1.3. If $f(\varrho, \beta)$ is a strictly convex function of $\varrho, 0<\varrho<1$ for a fixed $\beta$, then the gas has uniform density in the thermodynamic limit for this $\beta$, and any $\varrho$, $0<\varrho<1$.

Using this we can now show

Theorem 1.4. If $\alpha>1$ there is a $\beta_{1}$ such that if $\beta \leq \beta_{1}$ the gas has uniform density in the thermodynamic limit.

Proof. If we put $f(1, \beta)=\lim _{\varrho \rightarrow 1-} f(\varrho, \beta)$ and $f(\varrho, \beta)=+\infty$ for $\varrho \notin[0,1]$, then by the lattice analogue of Theorem 3.4.4 in Ruelle [8], $f(\varrho, \beta)$ is a lower semicontinuous convex function of $\varrho \in R$. By the lattice analogue of Theorem 3.4.6 in Ruelle [8] the pressure $p(\mu, \beta)$ as a function of the chemical potential $\mu$ is given by the Legendre-Fenchel transform

$$
\beta p(\mu, \beta)=\sup _{\varrho \in R}(\varrho \mu-\beta f(\varrho, \beta)) .
$$

Theorem 5.2.1 in Ruelle [8] says that there is a $\beta_{1}$ such that $p(\mu, \beta)$ is an analytic function of $\mu$ when $\beta \leq \beta_{1}$. By a theorem for convex functions, see Rockafellar [7] p. 253, $f(\varrho, \beta)$ is essentially strictly convex when $p(\mu, \beta)$ is differentiable as a function of $\mu$. Thus $f(\varrho, \beta)$ is strictly convex in $[0,1)$ where it is finite.

Corollary 1.5. If $\beta \geq \beta_{0}$, then $\partial p(\mu, \beta) / \partial \mu$ does not exist for all $\mu \in R$.

The lattice gas can be related to the Ising model by the substitution $n_{i}=\frac{1}{2}\left(s_{i}+1\right), s_{i}= \pm 1$. This gives the following relation between the pressure $p(\mu, \beta)$ for the lattice gas and the free energy $\psi(\beta, h)$ for the Ising model with interaction $J_{i j}=|i-j|^{-\alpha}$ and magnetic field $h$,

$$
\psi(\beta, h)=4 \beta p(2 h-4 \beta c, 4 \beta)-h+\beta c,
$$

where $c=2 \zeta(\alpha)$ and $\zeta(\alpha)$ is Riemanns $\zeta$-function. Thus, by Corollary 1.5 , when $\beta \geq \beta_{0} / 4,(\partial \psi / \partial h)(\beta, h)$ does not exist for all $h \in R$. It is known that this can only happen when $h=0$ and that we then have spontaneous magnetization, see Ellis [3], p. 105. The theorems above thus imply that the $1 / r^{\alpha}$-Ising model in one dimension has a phase transition when $\alpha \leq 2$.

\section{Blocks and Rearrangement}

\subsection{Definition of Blocks}

We now begin the proof of Theorem 1. Basic for our proof is the partition of configurations into blocks which alternate between having a high and a low density of particles. Let $1 \leq a<a^{\prime} \leq L+1$ be two integers and $\underline{n} \in Q$ a 
configuration. Then

$$
A=\left\langle a, a^{\prime}\right\rangle=\left(n_{a}, n_{a+1}, \ldots, n_{a^{\prime}-1}\right)
$$

is called a block in $\underline{n}$. $A$ is an o-block if $n_{a}=n_{a^{\prime}-1}=1$ and an e-block if $n_{a}=n_{a^{\prime}-1}=0$. The infinite block $\langle-\infty, a\rangle, n_{a}=1$, is always an $o$-block and $\langle a, \infty\rangle, n_{a}=0$ is always an $e$-block, due to the boundary conditions. The length of the block $A$ is $|A|=a^{\prime}-a$, the number of positions in the block. Two $o$-(e-)blocks $A=\left\langle a, a^{\prime}\right\rangle$ and $B=\left\langle a^{\prime}, a^{\prime \prime}\right\rangle$ can be joined to a new $o-(e-)$ block $A B=\left\langle a, a^{\prime \prime}\right\rangle$. A set of integers $\gamma=\left\{a_{1}, \ldots, a_{r}\right\}, 0 \leq a_{1}<a_{2}<\cdots<a_{r} \leq L+1$ defines a partition of a configuration $\underline{n}$ into blocks $\left\langle a_{k}, a_{k+1}\right\rangle, k=0, \ldots, r$, where $a_{0}=-\infty, a_{r+1}=\infty$. We will say that $\left\langle a_{k}, a_{k+1}\right\rangle$ is a block in $(\underline{n}, \gamma)$. The number of elements in $\gamma$ will be denoted $|\gamma|$.

Let $\delta$ be as in Theorem 1.2. The constants $K$ and $\kappa$ will be specified later.

Definition 2.1. Let $\gamma(\underline{n})$ define a partition of the configuration $\underline{n}$ into blocks. We will say that $(\underline{n}, \gamma(\underline{n}))$ has the density property if the blocks in $(\underline{n}, \gamma(\underline{n}))$ alternate between $o$ - and $e$-blocks and for each $o-\left(e\right.$-)block $A=\left\langle a, a^{\prime}\right\rangle$ in $(\underline{n}, \gamma(\underline{n}))$,

(i) $N(a, x)(\underline{n}) \geq 1+(1-\delta)(x-a) \quad(\leq \delta(x-a))$,

(ii) $N\left(x, a^{\prime}-1\right)(\underline{n}) \geq 1+(1-\delta)\left(a^{\prime}-1-x\right) \quad\left(\leq \delta\left(a^{\prime}-1-x\right)\right)$

if $a \leq x \leq a^{\prime}-1$.

Thus if a partition has the density property the $o$ - and $e$-blocks that it defines have a high, respectively low, density of particles.

We will now define a partition $\gamma_{1}(\underline{n})$ for every $\underline{n} \in Q_{1}$. Put

$$
u_{k}=2^{k-1}, \quad v_{k}=\frac{2}{\delta} 4^{k-1} .
$$

The blocks in $\left(\underline{n}, \gamma_{1}(\underline{n})\right)$ will be the basic building blocks in our argument. $\gamma_{1}(\underline{n})$ is defined inductively. Let

$$
\gamma^{(0)}(\underline{n})=\left\{i \in\{1, \ldots, L+1\} ;\left|n_{i}-n_{i-1}\right|=1\right\} .
$$

The blocks defined by $\gamma^{(0)}(\underline{n})$ alternate between $o$ - and $e$-blocks and in every block either all $n_{i}=1$ or all $n_{i}=0$. Suppose that $\gamma^{(k-1)}(\underline{n})$ has been defined and that the blocks defined by it alternate between $o$ - and $e$-blocks. Consider all $o$-blocks in $\left(\underline{n}, \gamma^{(k-1)}(\underline{n})\right)$ of length $\geq v_{k}, A_{j_{1}}, \ldots, A_{j_{p}}, p \geq 1,\left(\left\langle-\infty, a_{1}\right\rangle\right.$ is always an $o$-block of length $\left.\geq v_{k}\right)$. If the $e$-blocks in $\left(\underline{n}, \gamma^{(k-1)}(\underline{n})\right)$ between $A_{j_{r}}=\left\langle a_{j_{r}}, a_{j_{r}+1}\right\rangle$ and $A_{j_{r+1}}=\left\langle a_{j_{r+1}}, a_{j_{r+1}+1}\right\rangle$ have total length $\leq u_{k}$ we put $\eta_{r}=\left[a_{j_{r}+1}, a_{j_{r+1}}\right] \cap \gamma^{(k-1)}(\underline{n})$. If the $e$-blocks have length $>u_{k}$ we put $\eta_{r}=\emptyset$. Do this for $r=1, \ldots, p-1$; if $p=1$ there are no $\eta_{r}$ 's. In the same way we define $\lambda_{1}, \ldots, \lambda_{q-1}$ with $e$-blocks and $o$-blocks exchanged. Put

$$
\gamma^{(k)}(\underline{n})=\gamma^{(k-1)}(\underline{n}) \backslash\left(\eta_{1} \cup \cdots \cup \eta_{p-1} \cup \lambda_{1} \cup \cdots \cup \lambda_{q-1}\right) .
$$

It is clear from the construction that the blocks defined by $\gamma^{(k)}(\underline{n})$ alternate between $o$ - and $e$-blocks. We now let

$$
\gamma_{1}(\underline{n})=\gamma^{\left(2 k_{N}\right)}(\underline{n}),
$$

where

$$
k_{N}=\left[\frac{1}{4} \log N\right] .
$$

Lemma 2.2. Consider two o-(e-)blocks of length $\geq v_{k}$ in $\left(\underline{n}, \gamma_{1}(\underline{n})\right), \underline{n} \in Q_{1}$. Then the total length of the e-(o-)blocks between them is $>u_{k}, 1 \leq k \leq 2 k_{N}$. 
Proof. Assume that the lemma is false. Then there is a $k, 1 \leq k \leq 2 k_{N}$, and two $o$-(e-)blocks $\left\langle a, a^{\prime}\right\rangle$ and $\left\langle b, b^{\prime}\right\rangle$ in $\left(\underline{n}, \gamma_{1}(\underline{n})\right)$ of length $\geq v_{k}$, such that the length of the $e-(o-)$ blocks between them is $\leq u_{k}$ and all $o$ - $(e-)$ blocks between them have length $<v_{k}$. $a^{\prime}$ must have been a right endpoint of an $o-(e-)$ block $\left\langle a^{\prime \prime}, a^{\prime}\right\rangle$ and $b$ a left endpoint of an $o-(e-)$ block $\left\langle b, b^{\prime \prime}\right\rangle$ in $\left(\underline{n}, \gamma^{(k-1)}(\underline{n})\right)$. Since $b$ and $a^{\prime}$ were not removed at some later step the $e$ - $\left(o\right.$-)blocks between $\left\langle a^{\prime \prime}, a^{\prime}\right\rangle$ and $\left\langle b, b^{\prime \prime}\right\rangle$ in $\left(\underline{n}, \gamma^{(k-1)}(\underline{n})\right)$ have length $\leq u_{k}$ and one of the blocks $\left\langle a^{\prime \prime}, a^{\prime}\right\rangle$ and $\left\langle b, b^{\prime \prime}\right\rangle$, say $\left\langle a^{\prime \prime}, a^{\prime}\right\rangle$, must have length $<v_{k}$. But $a^{\prime \prime} \notin \gamma_{1}(\underline{n})$, and hence $a^{\prime \prime}$ must have been removed at some later step, whereas $a^{\prime}$ remained. This is only possible if $\left\langle a^{\prime \prime}, a^{\prime}\right\rangle$ has length $\geq v_{k}$ and we get a contradiction.

Lemma 2.3. $\left(\underline{n}, \gamma_{1}(\underline{n})\right)$ has the density property for every $\underline{n} \in Q_{1}$.

The proof of this lemma will be given in Sect.4.3.

\subsection{The Elementary Rearrangement}

Starting from a given configuration $\underline{n}$ and a partition $\gamma=\left\{a_{1}, \ldots, a_{2 r-1}\right\}$ of it, we will define a new configuration by letting two blocks in $(\underline{n}, \gamma)$ change place. $(\underline{n}, \gamma)$ consists of $2 r$ blocks and the elementary rearrangement operation, $S$, consists of letting the shortest block in $(\underline{n}, \gamma)$ change place with the shortest of its neighbouring blocks. To be more precise, let $S_{k, k+1}$ denote the operation where block number $k$ changes place with block number $k+1 . S_{k, k+1}$ maps a triple $(\underline{n}, \gamma, \delta)$ to a triple $\left(\underline{n}^{\prime}, \gamma^{\prime}, \delta^{\prime}\right)$, where $\underline{n}, \underline{n}^{\prime}$ are configurations, $\gamma, \gamma^{\prime}$ are partitions and $\delta, \delta^{\prime}$ are sets which give the positions of the "old partition points." We define

$$
\begin{aligned}
\underline{n}^{\prime}= & \left\langle a_{0}, a_{1}\right\rangle \ldots\left\langle a_{k-2}, a_{k-1}\right\rangle\left\langle a_{k}, a_{k+1}\right\rangle\left\langle a_{k-1}, a_{k}\right\rangle\left\langle a_{k+1}, a_{k+2}\right\rangle \ldots\left\langle a_{2 r-1}, a_{2 r}\right\rangle, \\
\gamma^{\prime}= & \left(\gamma \backslash\left\{a_{k-1}, a_{k}, a_{k+1}\right\}\right) \cup\left\{a_{k-1}+a_{k+1}-a_{k}\right\}, \\
\delta^{\prime}= & \left(\delta \backslash\left(\delta \cap\left[a_{k-1}, a_{k}\right)\right)\right) \cup\left(\delta \cap\left[a_{k-1}, a_{k}\right)+a_{k+1}-a_{k}\right) \\
& \cup\left(\delta \backslash\left(\delta \cap\left[a_{k}, a_{k+1}\right)\right) \cup\left(\delta \cap\left[a_{k}, a_{k+1}\right)-\left(a_{k}-a_{k-1}\right)\right) \cup\left\{a_{k-1}, a_{k+1}\right\} .\right.
\end{aligned}
$$

We can now define the elementary rearrangement operation $S$ as follows. If $\left\langle a_{k-1}, a_{k}\right\rangle$ is the shortest block in $(\underline{n}, \gamma)$, the leftmost if there are several, then $S=S_{k-1, k}$ if $\left|a_{k-1}-a_{k-2}\right| \leq\left|a_{k+1}-a_{k}\right|$ and $S=S_{k, k+1}$ if $\left|a_{k-1}-a_{k-2}\right|>\left|a_{k+1}-a_{k}\right|$. To motivate the definition of $\gamma^{\prime}$ and $\delta^{\prime}$, write $A_{k}=\left\langle a_{2(k-1)}, a_{2 k-1}\right\rangle, B_{k}=\left\langle a_{2 k-1}, a_{2 k}\right\rangle$, $k=1, \ldots, r$, and assume that $A_{p}$ is the shortest block and $\left|B_{p-1}\right| \leq\left|B_{p}\right|$. Then the partition points between $A_{p-1}, B_{p-1}$, between $B_{p-1}, A_{p}$ and between $A_{p}, B_{p}$ are removed from $\gamma$ and the new partition point between $A_{p-1} A_{p}$ and $B_{p-1} B_{p}$ is added to $\gamma$, giving $\gamma^{\prime}$. The "old partition points" tell us how $A_{p-1} A_{p}$ and $B_{p-1} B_{p}$ should be split up to give $A_{p-1}, A_{p}, B_{p-1}$ and $B_{p}$; they are added to $\delta$. We also have to move previous "old partition points" lying inside $B_{p-1}$ and $A_{p}$. This explains the definition of $\delta^{\prime}$. Compare Fig. 1.

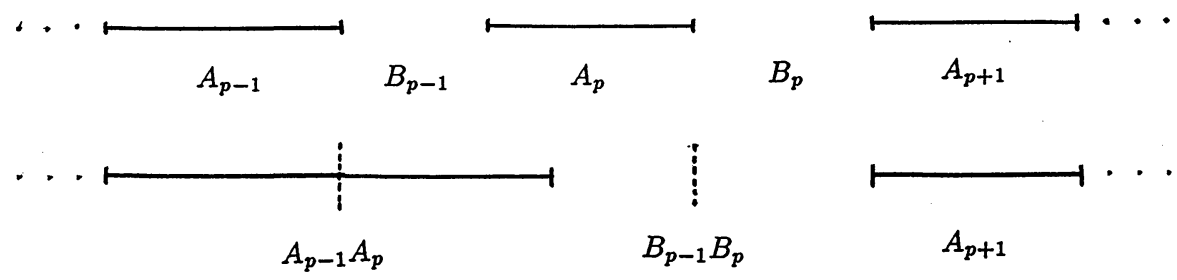

Fig. 1. The dotted lines indicate the locations of the old partition points which are added to $\delta$ 


\subsection{The Local Rearrangement}

Starting with $Q_{1}$ we will now by successive elementary rearrangements define sets of configurations $Q_{j}, j=1, \ldots, k_{N}$, and for each $\underline{n} \in Q_{j}$ a partition $\gamma_{j}(\underline{n})$. Assume that $Q_{j-1}$ has been defined and that each $\underline{m} \in \bar{Q}_{j-1}$ has been assigned a partition $\gamma_{j-1}(\underline{m})$. For each $\underline{m} \in Q_{j-1}$ we do successive elementary rearrangements until the shortest block is $\geq u_{j}$. This will surely happen after a finite number, say $l$, of elementary rearrangements, where $l$ of course depends on $\underline{m}$. Let

$$
(R(\underline{m}), R \gamma(\underline{m}), R \delta(\underline{m}))=S^{l}\left(\underline{m}, \gamma_{j-1}(\underline{m}), \emptyset\right) .
$$

We define $Q_{j}$ and $\gamma_{j}(\underline{n})$ as follows

$$
\begin{aligned}
Q_{j} & =\left\{R(\underline{m}) ; \underline{m} \in Q_{j-1}\right\}, \\
\gamma_{j}(\underline{n}) & =\bigcup_{\underline{m} \in Q_{j-1} ; R(\underline{m})=\underline{n}} R \gamma(\underline{m}) .
\end{aligned}
$$

Lemma 2.4. $\underline{m} \in Q_{j-1}, j \geq 2$, is uniquely determined by $(R(\underline{m}), R \gamma(\underline{m}), R \delta(\underline{m}))$.

Proof. It is clear that the blocks in $R(\underline{m})$ defined by $R \gamma(\underline{m})$ alternate between $o$ - and $e$-blocks. Let $R \gamma(\underline{m})=\left\{a_{1}, \ldots, a_{2 r-1}\right\}$ and $R \delta(\underline{m})=\left\{b_{1}, \ldots, b_{s}\right\}$. Then $A_{k}=\left\langle a_{2(k-1)}, a_{2 k-1}\right\rangle, k=1, \ldots, r$, are $o$-blocks and $B_{k}=\left\langle a_{2 k-1}, a_{2 k}\right\rangle, k=1, \ldots, r$, are $e$-blocks. $R \delta(\underline{m})$ tells us how to split these into smaller blocks, e.g. if $a_{2(k-1)}<b_{i}<b_{i+1}<\cdots<b_{i+p}<a_{2 k-1}$, then $A_{k}$ should be split up into $o$ blocks $\left\langle a_{2(k-1)}, b_{i}\right\rangle, \ldots,\left\langle b_{i+p}, a_{2 k-1}\right\rangle$. Similarly the other blocks may be split up into smaller blocks of the same type. The $o$ - and $e$-blocks thus obtained are the $o$ - and $e$-blocks in $\left(\underline{m}, \gamma_{j-1}(\underline{m})\right)$. These blocks are correctly ordered since in the rearrangement procedure we do not change the order of the $o$-blocks among themselves or the $e$-blocks among themselves. We thus know the $o$ - and $e$ blocks in $\left(\underline{m}, \gamma_{j-1}(\underline{m})\right)$ in the right order and this enables us to reconstruct $\underline{m}$ uniquely.

The proof of the following lemma is postponed to Sect.4.3.

Lemma 2.5. $\left(\underline{n}, \gamma_{j}(\underline{n})\right)$ has the density property for every $\underline{n} \in Q_{j}$ and all blocks in $\left(\underline{n}, \gamma_{j}(\underline{n})\right), \underline{n} \in Q_{j}$, have length $\geq u_{j}$.

\section{The Energy-Entropy Argument}

\subsection{The Entropy Lemma}

As a consequence of the definition of the original partition and the rearrangement procedure there is a bound on how much a block can grow due to the rearrangements. This gives an estimate of the maximal distance from an element in $R \delta(\underline{m})$ to the nearest element in $R \gamma(\underline{m})$. More precisely we have the following lemma.

Lemma 3.1. Let $w_{j-1}=9^{j-2} v_{j}$. Then for all $\underline{m} \in Q_{j-1}$ the distance from an element in $R \delta(\underline{m})$ to the closest element in $R \gamma(\underline{m})$ is $\leq 8 w_{j-1}, 2 \leq j \leq k_{N}$.

The proof of Lemma 3.1 is postponed to Sect.4. We can now estimate the entropy loss due to the rearrangement. 
Lemma 3.2. (The entropy lemma). Let $\underline{n} \in Q_{j}, 2 \leq j \leq k_{N}$ and let $1 \leq K \leq\left|\gamma_{j}(\underline{n})\right|$, $M \geq 0$ be integers. If $L_{K, M}(\underline{n})$ denotes the number of configurations $\underline{m} \in Q_{j-1}$ such that $R(\underline{m})=\underline{n},|R \gamma(\underline{m})|=K$ and $|R \delta(\underline{m})|=M$, then

$$
L_{K, M} \leq e^{C_{1} j M}\left(\begin{array}{c}
\left|\gamma_{j}(\underline{n})\right| \\
K
\end{array}\right) \sum_{r=0}^{\min (K, M)}\left(\begin{array}{c}
K \\
r
\end{array}\right)
$$

where $C_{1}=\log (72 / \delta)$.

Proof. By Lemma $2.2 \underline{m}$ is uniquely determined if we know $\underline{n}=R(\underline{m}), R \gamma(\underline{m})$ and $R \delta(\underline{m})$. It follows from (2.1) that the number of possible $R \gamma(\underline{m})$ with $|R \gamma(\underline{m})|=K$ is not more than

$$
\left(\begin{array}{c}
\left|\gamma_{j}(\underline{n})\right| \\
K
\end{array}\right)
$$

Imagine that each element in $R \delta(\underline{m})$ is assigned to the element in $R \gamma(\underline{m})$ which is closest to it. Suppose that $r$ elements in $R \gamma(\underline{m})$ have been assigned elements from $R \delta(\underline{m}), r \leq K, r \leq M$. There are $\left(\begin{array}{c}K \\ r\end{array}\right)$ possibilities for these $r$ elements. $R \delta(\underline{m})=\left\{b_{1}, \ldots, b_{M}\right\}, b_{1}<b_{2}<\cdots<b_{M}$, is split up into $r$ subsets $\left\{b_{1}, \ldots, b_{m_{1}}\right\}$, $\left\{b_{m_{1}+1}, \ldots, b_{m_{1}+m_{2}}\right\}, \ldots,\left\{b_{m_{1}+\cdots+m_{r-1}+1}, \ldots, b_{m_{1}+\cdots+m_{r}}\right\}, m_{1}+\cdots+m_{r}=M$. This can be done in $\left(\begin{array}{c}M \\ r-1\end{array}\right) \leq 2^{M}$ ways. By $(a)_{j}$ the distance from an element in $R \delta(\underline{m})$ to the element it has been assigned to is $\leq 8 w_{j-1}$. Thus the number of possibilities for the positions of the elements in $R \delta(\underline{m})$, given $m_{1}, \ldots, m_{r}$ and the elements in $R \gamma(\underline{m})$ they have been assigned to, is at most

$$
\prod_{i=1}^{r}\left(\begin{array}{c}
16 w_{j-1} \\
m_{i}
\end{array}\right) \leq e^{M \log \left(16 w_{J-1}\right)} \leq e^{j M \log (72 / \delta)}
$$

where we have used that $\left(\begin{array}{l}n \\ k\end{array}\right) \leq \exp (k \log n)$. Combining all the combinatorial
estimates we obtain (3.1).

\subsection{The Energy Lemma}

We also need an estimate of how much the energy changes when we perform an elementary rearrangement. This is provided by the next lemma which will be proved in Sect. 5 .

Lemma 3.3. (The energy lemma). Let $(\underline{n}, \gamma)$ satisfy the density property and assume that all blocks in $(\underline{n}, \gamma)$ have length $\geq 2^{j-1}$. If $\underline{n}^{\prime}$ is obtained from $\underline{n}$ by an elementary rearrangement of $(\underline{n}, \gamma)$, then

$$
H(\underline{n})-H\left(\underline{n}^{\prime}\right) \geq 2 \kappa j,
$$

where $\kappa$ is a positive constant that only depends on $\alpha$.

Remark. When $\alpha<2$ the right-hand side of (3.2) can be replaced by $\kappa^{\prime} 2^{(2-\alpha)(j-1)}$.

From the energy lemma it follows that for all $\underline{m} \in Q_{j-1}, 2 \leq j \leq k_{N}$ we have

$$
H(\underline{m})-H(R \underline{m})) \geq \kappa|R \delta(\underline{m})| j .
$$

To see this note that the partitions we obtain in the intermediate steps as we go from $\underline{m}$ to $T(\underline{m})$ by successive elementary rearrangements all satisfy the density 
property. Thus we can use Lemma 3.2 repeatedly and since the total number of elementary rearrangements we do is $|R \delta(\underline{m})| / 2$ and all blocks have length $\geq 2^{j-1}$ by Lemma 2.5 we get (3.3).

We can now specify the constants appearing in Theorem 1.2. Let

$$
\left.\delta_{0}=\min \{\varrho, 1-\varrho, 1 / 4,2(\alpha-1) \kappa / 5 \log 2)\right\}
$$

and

$$
\beta_{0}=\left(3+\log \left(72 / \delta_{0}\right)\right) / \kappa .
$$

For a given $\beta \geq \beta_{0}$ we let $\delta$ be given by

$$
\beta=(3+\log (72 / \delta)) / \kappa,
$$

i.e. $\delta=K \exp (-\kappa \beta)$, where $K$ is a numerical constant. Note that $\beta \geq \beta_{0}$ implies $\delta \leq \delta_{0}$.

\subsection{Energy-Entropy Argument for the Local Rearrangement}

Above we have defined maps $R: Q_{j-1} \rightarrow Q_{j}, j=2, \ldots, k_{N}$ and together they define a map $\mathscr{R}: Q_{1} \rightarrow Q_{k_{N}}$. Define

$$
H_{1}=\left\{\underline{n} \in Q_{1} ;\left|\gamma_{k_{N}}(\mathscr{R}(\underline{n}))\right| \geq 3\right\}
$$

and $H_{j}=R\left(H_{j-1}\right)$. Note that $H_{j} \subset Q_{j}$ so we have a partition $\gamma_{j}(\underline{n})$ for each $\underline{n} \in H_{j}$. We shall prove that

$$
\lim _{\Lambda \rightarrow Z^{+}} P\left(H_{1}\right)=0 .
$$

According to the next lemma, which will be proved in Sect.4.2, this proves Theorem 1.2.

Lemma 3.4. Let $d_{1}, d_{2}$ be defined by (1.6). If $0 \leq \tau_{1}<\tau_{2} \leq d_{1}-\varepsilon, \varepsilon>0$, then

$$
\left\{\underline{n} \in Q ; d\left(\tau_{1}, \tau_{2}\right)(\underline{n})<1-2 \delta\right\} \subset H_{1}
$$

and if $d_{2}+\varepsilon \leq \tau_{1}<\tau_{2} \leq 1$, then

$$
\left\{\underline{n} \in Q ; d\left(\tau_{1}, \tau_{2}\right)(\underline{n})>2 \delta\right\} \subset H_{1}
$$

for all sufficiently large $N, L$.

We now turn to the proof of (3.5). The following inequality controls the energy-entropy balance in the local rearrangements. For $1 \leq k \leq k_{N}$ we have

$$
\sum_{\underline{m} \in H_{1}} e^{-\beta H(\underline{n})} \leq \eta_{k} \sum_{\underline{m} \in H_{k}} e^{-\beta H(\underline{m})+\log (2 k)\left|\gamma_{k}(\underline{m})\right|},
$$

where $\eta_{k} \leq \eta$ and $\eta$ is a numerical constant. We will prove (3.6) by induction. Assume that (3.6) is true for $k=j-1$. Let $D_{j-1}=\log 2(j-1)$. Then using Lemma 3.2 and (3.3) we find

$$
\begin{aligned}
\sum_{\underline{m} \in H_{j-1}} e^{-\beta H(\underline{m})+D_{j-1}\left|\gamma_{j-1}(\underline{m})\right|} & =\sum_{\underline{n} \in H_{j}} e^{-\beta H(\underline{n})} \sum_{\underline{m} \in H_{j-1} ; R(\underline{m})=\underline{\underline{n}}} e^{-\beta\left(H(\underline{m})-H(R(\underline{m}))+D_{j-1}\left|\gamma_{j-1}(\underline{m})\right|\right.} \\
& \leq \sum_{\underline{n} \in H_{j}} e^{-\beta H(\underline{n})} \sum_{K=1}^{\left|\gamma_{j}(\underline{\underline{n}})\right|} \sum_{M=0}^{\infty} e^{-\beta K M j+D_{j-1}(M+K)} L_{K, M}(\underline{n}),
\end{aligned}
$$


where we have also used that $\left|\gamma_{j-1}(\underline{m})\right|=|R \gamma(\underline{m})|+|R \delta(\underline{m})|$, which follows from the fact that in every elementary rearrangement, $S(\underline{n}, \gamma, \delta)=\left(\underline{n}^{\prime}, \gamma^{\prime}, \delta^{\prime}\right),\left|\gamma^{\prime}\right|=|\gamma|-2$, $\left|\delta^{\prime}\right|=|\delta|+2$, and hence $|\gamma|+|\delta|=\left|\gamma^{\prime}\right|+\left|\delta^{\prime}\right|$. Using (3.1) we see that (3.7) can be estimated by

$$
\sum_{\underline{n} \in H_{j}} e^{-\beta H(\underline{n})} \sum_{K=1}^{\left|\gamma_{j}(\underline{n})\right|} e^{D_{j-1} K}\left(\begin{array}{c}
\left|\gamma_{j}(\underline{n})\right| \\
K
\end{array}\right) G(K, \underline{n})
$$

where

$$
G(K, \underline{n})=\sum_{M=0}^{\infty} \sum_{r=0}^{\min (K, M)}\left(\begin{array}{c}
K \\
r
\end{array}\right) e^{-\left(\beta \kappa-C_{1}\right) j M} .
$$

According to (3.4) $\beta \kappa-C_{1}=3 \geq 1$. Thus

$$
\begin{aligned}
G(K, \underline{n}) & =\left(\sum_{M=0}^{K} \sum_{r=0}^{M}+\sum_{M=K+1}^{\infty} \sum_{r=0}^{K}\right)\left(\begin{array}{c}
K \\
r
\end{array}\right) e^{-j M} \\
& \geq \sum_{r=0}^{K}\left(\begin{array}{c}
K \\
r
\end{array}\right) \sum_{M=0}^{K} e^{-j M}+\sum_{M=K}^{\infty} 2^{K} e^{-j M} \\
& \leq \frac{1}{1-e^{-j}}\left[\left(1+e^{-j}\right)^{K}+2 e^{-j K}\right] \\
& \leq \frac{1+2 e^{-j}}{1-e^{-j}}\left(1+e^{-j}\right)^{K} .
\end{aligned}
$$

Inserting this in (3.8) and calculating the sum over $K$ using the binomial theorem the expression in (3.8) can be estimated by

$$
\left(1+2 e^{-j}\right)^{2} \sum_{\underline{n} \in H_{j}} e^{-\beta H(\underline{n})}\left(1+e^{D_{j-1}}\left(1+e^{-j}\right)\right)^{\left|\gamma_{j}(\underline{n})\right|} .
$$

Since $\log \left(1+e^{D_{j-1}}\left(1+e^{-j}\right)\right) \leq \log (2 j)$ we have completed the induction step if we put $\eta_{j}=\eta_{j-1}\left(1+2 e^{-j}\right)^{2}$. We see that

$$
\left.\eta_{k}=\prod_{j=2}^{k} 1+2 e^{-j}\right)^{2} \leq \prod_{j=1}^{\infty}\left(1+2 e^{-j}\right)^{2}=\eta,
$$

and (3.6) is proved.

\subsection{Energy-Entropy Argument for the Global Rearrangement}

We are now in position to rigorously carry out the argument sketched in the introduction. In $H_{k_{N}}$ all blocks have length $\geq 2^{k_{N}-1} \sim N^{(\log 2) / 4}$ and this is sufficient for that argument to work. For each $\underline{m} \in H_{k_{N}}$ we will perform successive elementary rearrangements starting from $\left(\underline{m}, \gamma_{k_{N}}(\underline{m}), \emptyset\right)$. Every elementary rearrangement reduces the number of elements in the partition with 2 . We apply the $S$-operations repeatedly until we get a configuration $(G(\underline{m}), G \gamma(\underline{m}), G \delta(\underline{m}))$ with only one partition point, i.e. $|G \gamma(\underline{m})|=1$. Just as in Lemma 2.2 we see that $\underline{m}$ is uniquely determined by $(G(\underline{m}), G \gamma(\underline{m}), G \delta(\underline{m}))$. It is clear by the rearrangement procedure that $|G \gamma(\underline{m})|+|G \delta(\underline{m})|=\left|\gamma_{k_{N}}(\underline{m})\right| \cdot\left|\gamma_{k_{N}}(\underline{m})\right|=2 M-1$ is odd and, by the 
definition of $H_{1}, M \geq 2$. Given $G(\underline{m})$ the number of possibilities for $G \gamma(\underline{m})$ and $G \delta(\underline{m})$ is less than

$$
L\left(\begin{array}{c}
L \\
2 M-2
\end{array}\right) \leq e^{(2 M-1) \log L} .
$$

When we go from $\underline{m}$ to $G(\underline{m})$ we do $|G \delta(\underline{m})| / 2=M-1$ elementary rearrangements. Repeated application of Lemma 3.2 gives

$$
H(\underline{m})-H(G(\underline{m})) \geq 2 \kappa(M-1) k_{N}
$$

since all blocks in $H_{k_{N}}$ have length $\geq 2^{k_{N}-1}$.

Thus by (3.6) with $k=k_{N}$, (3.9) and (3.10) we obtain

$$
\begin{aligned}
P\left(H_{1}\right) & \leq \frac{\eta}{Z} \sum_{\underline{n} \in G\left(H_{k_{N}}\right)} e^{-\beta H(\underline{n})} \sum_{M=2}^{\infty} e^{\log \left(2 k_{N} L\right)(2 M-1)-2 \beta \kappa(M-1) k_{N}} \\
& \leq \eta e^{\log \left(2 k_{N} L\right)} \sum_{M=1}^{\infty} e^{\left(2 \log \left(2 k_{N} L\right)-\frac{1}{2} \beta \kappa \log N\right) M} \leq C \frac{(\log N)^{3}}{N}
\end{aligned}
$$

for all sufficiently large $N, L$. Here we have used that $\beta \kappa \geq \beta_{0} \kappa \geq 8$. It follows from (3.11) that (3.5) holds and we have proved Theorem 1.2.

\section{Proof of the Entropy Lemma}

\subsection{Proof of Lemma 3.1}

Call the assertions in Lemma $3.1(a)_{j}$ for a particular value of $j$. Let

$$
w_{j, k}=9^{j-1} v_{k}, \quad w_{j-1}=w_{j-1, j} .
$$

Together with $(a)_{j}$ we will prove the following assertion which we call $(b)_{j}$, $2 \leq j \leq k_{N}$.

Consider two o-(e-)blocks in $\left(\underline{n}, \gamma_{j}(\underline{n})\right), \underline{n} \in Q_{j}$, of length $\geq w_{j, k}$. Then the total length of the e-(o-)blocks between them is $\geq u_{k}, j \leq k \leq 2 k_{N}-j$.

From Lemma 2.2 we know that $(b)_{1}$ is true. We will prove $(a)_{j}$ inductively by showing that $(b)_{j-1}$ implies $(a)_{j}$ and that $(b)_{j-1}$ and $(a)_{j}$ together imply $(b)_{j}$.

Assume that $(b)_{j-1}$ is true. $(R(\underline{m}), R \gamma(\underline{m}))$ has been obtained from $\left(\underline{m}, \gamma_{j-1}(\underline{m})\right)$ by successive elementary rearrangements. Consider a block $A$ in $(R(\underline{m}), R \gamma(\underline{m}))$. Since there is a symmetry between $o$ - and $e$-blocks we can assume that $A$ is an $o$-block. $A$ has been built up from $o$-blocks $A_{1}, \ldots, A_{r}$ in $\left(\underline{m}, \gamma_{j-1}(\underline{m})\right)$. If we can show that

$$
\min _{1 \leq p \leq r} \max \left\{\left|A_{1} \ldots A_{p-1}\right|,\left|A_{p+1} \ldots A_{r}\right|\right\} \leq 8 w_{j-1}
$$

then $(a)_{j}$ follows. In $\left(\underline{m}, \gamma_{j-1}(\underline{m})\right)$ we had $e$-blocks $B_{1}, \ldots, B_{r-1}$ between $A_{1}, \ldots, A_{r}$,

$$
\underline{m}=\ldots B_{0} A_{1} B_{1} A_{2} B_{2} \ldots A_{r-1} B_{r-1} A_{r} B_{r} \ldots
$$

$R(\underline{m})=\ldots B A B^{\prime} \ldots$, where $B=\ldots B_{0} \ldots B_{s-1}$ and $B^{\prime}=B_{s} \ldots B_{r} \ldots$ for some $s$, $1 \leq s \leq r$. It follows that $A_{s}$ must have been fixed throughout the rearrangement procedure, since if $A_{s}$ has been involved in a rearrangement $B_{s-1}$ and $B_{s}$ would have been joined. 
Claim 1. If the elementary rearrangement $\ldots A^{1} B^{1} A^{2} B^{2} \ldots$ to $\ldots A^{1} A^{2} B^{1} B^{2} \ldots$ is performed at some step, then $\left|A^{2}\right| \leq\left|A^{1}\right|$ and $\left|B^{1}\right| \leq\left|B^{2}\right|$, and one of $B^{1}$ and $A^{2}$ has length $<u_{j}$. Here $A^{1}$ can be an o- or an e-block.

This follows immediately from the definition of an elementary rearrangement.

Claim 2. Consider an e-block, $B^{p}$, to the left (right) of the o-block containing $A_{s}$ at some step in the rearrangement procedure. Let $A^{1}, \ldots, A^{p}$ be the o-blocks built up from $A_{1}, \ldots A_{r}$ to the left (right) of $B^{p}$. If $\left|B^{p}\right| \geq u_{j}$, then $\left|A^{j}\right|<u_{j}, j=1, \ldots, p$ and $\left|A^{1} \ldots A^{p}\right| \leq 3 w_{j-1}$.

This is seen as follows. We have the configuration

$$
\ldots A^{1} B^{1} \ldots A^{p} B^{p} A^{p+1} \ldots
$$

where $B^{1}, \ldots, B^{p}$ are built up from some (or all) of $B_{1}, \ldots, B_{s-1}$. Assume that $\left|A^{q}\right| \geq u_{j}$, some $q, 1 \leq q \leq p$. Because $B^{p}$ eventually ends up in $B$, at some step an $e$-block containing $B^{p}$ must change place with an $o$-block containing $A^{q}$, but this is impossible according to Claim 1 since they both have length $\geq u_{j}$. Thus $\left|A^{q}\right|<u_{j}, 1 \leq q \leq p$, and (4.2) actually looks like

$$
\ldots A_{1} B_{1} A_{2} \ldots A_{p} B^{p} A^{p+1} \ldots
$$

i.e. $A^{i}=A_{i}, i=1, \ldots, p$.

If some $o$-block $A_{i}$ is joined with $A_{i-1}$ before it is joined with $A_{i+1}$ (or $A^{p+1} \ldots$ ) we get an $o$-block of length $\geq u_{j}$ to the left of $B^{p}$ and a similar contradiction. Thus $A_{p}, \ldots, A_{1}$ are joined successively to the $o$-block containing $A^{p+1}$. It follows from Claim 1 that when $A_{3}$ is joined with the $o$-block containing $A_{4}$ we must have

$$
\left|B_{2}\right| \geq\left|B_{2} \ldots B_{p}\right| \text {. }
$$

Similarly we must have

$$
\left|B_{1}\right| \geq\left|B_{2} \ldots B_{p}\right| \text {. }
$$

If $\left|B_{2}\right| \geq w_{j-1}$, then $\left|B_{1}\right| \geq w_{j-1}$ and by $(b)_{j-1},\left|A_{2}\right| \geq u_{j}$ a contradiction. Hence $\left|B_{2}\right|<w_{j-1}$ and by (4.3)

$$
w_{j-1} \geq\left|B_{3} \ldots B_{p}\right| \geq(p-2) u_{j-1},
$$

and consequently

$$
\left|A_{1} \ldots A_{p}\right| \leq p u_{j} \leq\left(w_{j-1} / u_{j-1}+2\right) u_{j} \leq 3 w_{j-1} .
$$

Claim 3. Let $t \leq s$. If $A_{t-1}$ is joined with $A_{t}$ before $A_{t-2}$ is joined with $A_{t-1}$, then $\left|A_{1} \ldots A_{t-2}\right| \leq 3 w_{j-1}$. Similarly for $t \geq s$, if $A_{t+1}$ is joined with $A_{t}$ before $A_{t+2}$ is joined with $A_{t+1}$, then $\left|A_{t+2} \ldots A_{r}\right| \leq 3 w_{j-1}$.

This follows immediately from Claim 2, since when $A_{t-1}$ is joined with $A_{t}$ we get an $e$-block of length $\geq u_{j}$ either to the left or to the right of the $o$ block containing $A_{t-1}$. Now $A_{t-2}$ must lie to the left of this $e$-block and hence $\left|A_{1} \ldots A_{t-2}\right| \leq 3 w_{j-1}$.

We can now prove (4.1) by proving that $\max \left\{\left|A_{1} \ldots A_{s-1}\right|,\left|A_{s+1} \ldots A_{r}\right|\right\} \leq$ $8 w_{j-1}$. Assume first that $\left|A_{s}\right| \geq w_{j-1}$. If $\left|B_{s}\right| \geq u_{j}$, then $\left|A_{1} \ldots A_{s-1}\right| \leq 3 w_{j-1}$ by Claim 2. Suppose that $\left|B_{s-1}\right|<u_{j}$. Then $(b)_{j-1}$ implies that $\left|A_{s-1}\right|<w_{j-1}$. If $A_{s-1}$ and $A_{s}$ are joined before $A_{s-2}$ and $A_{s-1}$, then by Claim $3,\left|A_{1} \ldots A_{s-2}\right| \leq 3 w_{j-1}$, and hence $\left|A_{1} \ldots A_{s-1}\right| \leq w_{j-1}$. Otherwise, at some step an $o$-block $A_{s-j_{1}} \ldots A_{s-2}$, 
$j_{1} \geq 2$, is joined with $A_{s-1}$ which is not joined with $A_{s}$. If it is $A_{s-1}$ that changes place we get an $e$-block of length $\geq u_{j}$ to the right of $A_{s-1}$ and hence $\left|A_{1} \ldots A_{s-1}\right| \leq 3 w_{j-1}$. In the other case we get $\left|A_{s-j_{1}} \ldots A_{s-2}\right| \leq\left|A_{s-1}\right| \leq w_{j-1}$ and according to Claim $3,\left|A_{1} \ldots A_{s-j_{1}}\right| \leq w_{j-1}$. Thus $\left|A_{1} \ldots A_{s-1}\right| \leq 3 w_{j-1}$. By a symmetric argument we get $\left|A_{s+1} \ldots A_{r}\right| \leq 4 w_{j-1}$.

Now assume that $\left|A_{s}\right|<w_{j-1}$ and that $A_{s-1}$ is joined with $A_{s}$ before $A_{s+1}$ is joined with $A_{s}$, the other case is analogous. At some step an $o$-block $A_{j_{1}} \ldots A_{s-1}$ is joined with $A_{s}$ and by Claim $1,\left|A_{j_{1}} \ldots A_{s-1}\right| \leq\left|A_{s}\right|<w_{j-1}$. By Claim 3, $\left|A_{1} \ldots A_{j_{1}-1}\right| \leq 3 w_{j-1}$ and hence $\left|A_{1} \ldots A_{s-1}\right| \leq 4 w_{j-1}$. At some later step an $o$-block $A_{s+1} \ldots A_{k_{1}}$ is joined with an $o$-block $A_{j_{2}} \ldots A_{s}$. It follows from Claim 1 that $\left|A_{s+1} \ldots A_{k-1}\right| \leq\left|A_{j_{2}} \ldots A_{s}\right| \leq 5 w_{j-1}$. By Claim $3,\left|A_{k_{1}+1} \ldots A_{r}\right| \leq 3 w_{j-1}$ and thus $\left|A_{s+1} \ldots A_{r}\right| \leq 8 w_{j-1}$. This concludes the proof of $(a)_{j}$.

We now turn to the proof that $(b)_{j-1}$ and $(a)_{j}$ imply $(b)_{j}$. Assume that $(b)_{j}$ is false. Then there is an $\underline{n} \in Q_{j}$ and two $e$-blocks, say, $B$ and $B^{\prime}$ in $\left(\underline{n}, \gamma_{j}(\underline{n})\right)$ of length $\geq w_{j, k}$ such that the length of the $o$-blocks between $B$ and $B^{\prime}$ is $<u_{k}$, $k \geq j$. We must have $k \geq j+1$ since all blocks in $\left(\underline{n}, \gamma_{j}(\underline{n})\right)$ have length $\geq u_{j}$. Let $A_{1}, \ldots, A_{p}$ be the $o$-blocks between $B=\left\langle b_{0}, a_{1}\right\rangle$ and $B^{\prime}=\left\langle b_{p}, a_{p+1}\right\rangle$. Write $A_{i}=\left\langle a_{i}, b_{i}\right\rangle$ and $B_{i}=\left\langle b_{i}, a_{i+1}\right\rangle$. By (2.1) there is an $\underline{m} \in Q_{j-1}, R(\underline{m})=\underline{n}$ such that $a_{1} \in R \gamma(\underline{m})$. It follows from the density property that at least one of the $b_{i}^{\prime} s$ must belong to $R \gamma(\underline{m})$, and we let $b_{q}$ be the largest such $b_{i}$. In $(R(\underline{m}), R \gamma(\underline{m}))$ we have two $e$-blocks $C=\left\langle c, a_{1}\right\rangle$, where $c \leq b_{0}$ and $C^{\prime}=\left\langle b_{q}, c^{\prime}\right\rangle$, where $c^{\prime} \geq a_{p+1}$. Hence $C$ and $C^{\prime}$ have length $\geq w_{j, k}$. Consider an $o$-block $A$ in $(R(\underline{m}), R \gamma(\underline{m}))$ between $C$ and $C^{\prime}$. Then $A=\left\langle a_{r}, b_{s}\right\rangle$ for some $r, s, 1 \leq r \leq s \leq q$. If $r=s$, then $|A|=\left|A_{r}\right|$. Assume that $r<s$. Then $A=A_{r} B_{r} \ldots B_{s-1} A_{s}$. From the density property for $(R(\underline{m}), R \gamma(\underline{m}))$ we get that the number of occupied positions in $A$ is

$$
\geq(1-\delta)|A| \geq(1-\delta)\left(\left|A_{r}\right|+\left|B_{r}\right|+\cdots+\left|B_{s-1}\right|+\left|A_{s}\right|\right) .
$$

On the other hand we get from the density property for $\left(\underline{n}, \gamma_{j}(\underline{n})\right)$ that the number of occupied positions in $A$ is

$$
\leq\left|A_{r}\right|+\cdots+\left|A_{s}\right|+\delta\left(\left|B_{r}\right|+\cdots+\left|B_{s-1}\right|\right) .
$$

It follows that

$$
|A| \leq\left(1+\frac{\delta}{1-2 \delta}\right)\left(\left|A_{r}\right|+\cdots+\left|A_{s}\right|\right)
$$

We have thus shown that the total length of the $o$-blocks in $(R(\underline{m}), R \gamma(\underline{m}))$ between $C$ and $C^{\prime}$ is $\leq\left(1+\delta(1-2 \delta)^{-1}\right) \leq 2$ times the length of the $o$-blocks in $\left(\underline{n}, \gamma_{j}(\underline{n})\right)$ between $B$ and $B^{\prime}$, i.e. $<2 u_{k}=u_{k+1}$.

We will need the following two inequalities which are easily proved. If $k \geq j+1$, then

$$
w_{j, k}>32 w_{j-1} \quad \text { and } \quad w_{j, k}-16 w_{j-1}>w_{j-1, k+1} .
$$

It follows from $(a)_{j}$ that there must have been $e$-blocks $C_{1}$ and $C_{1}^{\prime}$ in $\left(\underline{m}, \gamma_{j-1}(\underline{m})\right)$ of length $\geq w_{j, k}-16 w_{j-1}>w_{j-1, k+1}$ which are part of $C$ respectively $C^{\prime}$. The $o-$ blocks in $(R(\underline{m}), R \gamma(\underline{m}))$ between $C$ and $C^{\prime}$ must have been built up from $o$-blocks in $\left(\underline{m}, \gamma_{j-1}(\underline{m})\right)$ between $C_{1}$ and $C_{1}^{\prime}$ because if an $o$-block is moved across $C_{1}$ or $C_{1}^{\prime}$ then they must at least double their length. Since $w_{j, k}-16 w_{j-1}>16 w_{j-1}$ this contradicts $(a)_{j}$. We thus have two $e$-blocks $C_{1}$ and $C_{1}^{\prime}$ of length $\geq w_{j-1, k+1}$ in $\left(\underline{m}, \gamma_{j-1}(\underline{m})\right)$, such that the total length of the $o$-blocks between them is $<u_{k+1}$. This contradicts $(b)_{j-1}$ if $k+1 \leq 2 k_{N}-(j-1)$, i.e. $k \leq 2 k_{N}-j$. 


\subsection{Proof of Lemma 3.4}

We will need the following lemma, which we will prove in Sect.4.3.

Lemma 4.1. Let $\gamma_{1}(\underline{n})=\left\{a_{1}, \ldots, a_{r}\right\}, \underline{n} \in Q_{1}$. If $0<x<x+z<a_{1}$ and $N(x, x+z)(\underline{n}) \leq(1-2 \delta) z$, then $x+L \geq C_{\delta} z^{2}$, where $C_{\delta}$ is a constant that only depends on $\delta$. Similarly, if $x+z>x>a_{r}$ and $N(x, x+z)(\underline{n}) \geq 2 \delta z$, then $x-a_{r} \geq C_{\delta} z^{2}$.

Let $\underline{n} \in Q_{1} \backslash H_{1}$ and let $\underline{m}=\mathscr{R}(\underline{n}) \in Q_{k_{N}}$. Then $\gamma_{k_{N}}(\underline{m})$ only contains one element which we denote by $\lambda$. Define

$$
Q_{j}(\underline{m})=\left\{\underline{n} \in Q_{j} ; R^{k_{N}-j}(\underline{n})=\underline{m}\right\} .
$$

Then $\underline{n} \in Q_{1}(\underline{m}), Q_{k_{N}}(\underline{m})=\{\underline{m}\}$ and $R\left(Q_{j-1}(\underline{m})\right)=Q_{j}(\underline{m})$. In $\left(\underline{m}, \gamma_{k_{N}}(\underline{m})\right),\langle-\infty, \lambda\rangle$ is an $o$-block and $\langle\lambda, \infty\rangle$ is an $e$-block. It follows from the density property that

$$
N=N(1, \lambda-1)(\underline{m})+N(\lambda, L)(\underline{m}) \leq \lambda+\delta(L-\lambda)
$$

and

$$
N=N(1, \lambda-1)(\underline{m})+N(\lambda, L)(\underline{m}) \geq(1-\delta) \lambda .
$$

Thus

$$
(1-\delta)^{-1}(N / L-\delta) \leq \lambda / L \leq(1-\delta)^{-1} N / L .
$$

Given $\varepsilon>0$ we see that for sufficiently large $N, L$,

$$
d_{1}-\varepsilon / 2 \leq \lambda / L \leq d_{2}+\varepsilon / 2,
$$

where $d_{1}$ and $d_{2}$ are given by (1.6). Define

$$
\begin{aligned}
v_{j} & =\min _{\underline{n} \in Q_{j}(\underline{m})}\left(\min \gamma_{j}(\underline{n})\right), \quad j=1, \ldots, k_{N}, \\
\mu_{j} & =\max _{\underline{n} \in Q_{j}(\underline{m})}\left(\max \gamma_{j}(\underline{n})\right), \quad j=1, \ldots, k_{N} .
\end{aligned}
$$

Let $\underline{n} \in Q_{j}(\underline{m})$. Then $\min \gamma_{j}(\underline{n})=\min R \gamma\left(\underline{n}^{\prime}\right)$ for some $\underline{n}^{\prime} \in Q_{j-1}(\underline{m})$ such that $R\left(\underline{n}^{\prime}\right)=\underline{n}$. It is clear from the definition of an elementary rearrangement that $\min R \gamma\left(\underline{n}^{\prime}\right) \geq \min \gamma_{j-1}\left(\underline{n}^{\prime}\right) \geq v_{j-1}$. Thus $v_{j} \leq v_{j-1}$. There is an $\underline{n}^{\prime \prime} \in Q_{j-1}(\underline{m})$ such that $v_{j-1}=\min \gamma_{j-1}\left(\underline{n}^{\prime \prime}\right)$. It follows from Lemma 3.1 that

$$
\min R \gamma\left(\underline{n}^{\prime \prime}\right)-\min \gamma_{j-1}\left(\underline{n}^{\prime \prime}\right) \leq 8 w_{j-1} .
$$

Furthermore $\min R \gamma\left(\underline{n}^{\prime \prime}\right) \geq \min \gamma_{j}\left(R\left(\underline{n}^{\prime \prime}\right)\right) \geq v_{j}$ since $R\left(\underline{n}^{\prime \prime}\right) \in Q_{j}(\underline{m})$. Thus $v_{j}-$ $v_{j-1} \leq 8 w_{j-1}$ and we obtain

$$
\lambda-v_{1}=v_{k_{N}}-v_{1} \leq \sum_{j=2}^{k_{N}} w_{j-1} \leq C N^{\gamma},
$$

where $C$ is a constant and $\gamma=\frac{1}{4} \log 36<1$. Similarly we can show that $\mu_{1}-\lambda \leq C N^{\gamma}$. Hence for $N, L$ sufficiently large,

$$
d_{1}-\varepsilon<\frac{v_{1}}{L} \leq \frac{\mu_{1}}{L} \leq d_{2}+\varepsilon .
$$

If $0 \leq \tau_{1} \leq \tau_{2} \leq d_{2}-\varepsilon$ and $\underline{n} \in Q_{1} \backslash H_{1}$ then $\left\langle\left[\tau_{1} L\right]\right.$, $\left.\left[\tau_{2} L\right]\right\rangle$ lies inside an $o$ block in $\left(\underline{n}, \gamma_{1}(\underline{n})\right)$. It follows from Lemma 4.1 that if $d\left(\tau_{1}, \tau_{2}\right)(\underline{n}) \leq 1-2 \delta$, then $\left[\tau_{1} L\right]+L \geq C_{\delta}\left(\left[\tau_{2} L\right]-\left[\tau_{1} L\right]\right)^{2}$, which is impossible if $L$ is sufficiently large. Hence $d\left(\tau_{1}, \tau_{2}\right)(\underline{n})>1-2 \delta$. Similarly, if $d_{2}+\varepsilon \leq \tau_{1}<\tau_{2} \leq 1$, then $\left\langle\left[\tau_{1} L\right],\left[\tau_{1} L\right]\right\rangle$ is part of an $e$-block in $\left(\underline{n}, \gamma_{1}(\underline{n})\right)$ and Lemma 4.1 implies that we must have $d\left(\tau_{1}, \tau_{2}\right)(\underline{n}) \leq 2 \delta$. This completes the proof of Lemma 3.4. 


\subsection{Proof of Lemmas 2.3, 2.5, and 4.1}

Proof of Lemma 2.2. The proof of (i) and (ii) in Definition 2.1 for $o$ - and $e$-blocks are similar and we will only give the proof of (i) for $o$-blocks. Let $\gamma^{(0)}, \ldots, \gamma^{\left(2 k_{N}\right)}=\gamma_{1}$ be the partitions used in the inductive definition of $\gamma_{1}$. Suppose that every $o$-block $A=\left\langle a, a^{\prime}\right\rangle$ in $\gamma^{(k-1)}(\underline{n})$ satisfies $N(a, a+t) \geq 1+\left(1-\delta_{k-1}\right) t$ for $0 \leq t \leq a^{\prime}-a$ and some $\delta_{k-1} \geq 0$ independent of $A$. This is obviously true for $k=1$ with $\delta_{0}=0$. Consider an $o$-block $B=\left\langle b, b^{\prime}\right\rangle$ defined by $\gamma^{(k)}(\underline{n})$. Assume that the length of whole or parts of $e$ - and $o$-blocks in $\left(\underline{n}, \gamma^{(k-1)}(\underline{n})\right)$ between $b$ and $b+s<b^{\prime}$ is $l$ and $s-l$ respectively. The $o$-blocks between $b$ and $b+s$ contain at least $1+\left(1-\delta_{k-1}\right)(s-l)$ particles according to our assumption. The construction of $\gamma^{(k)}(\underline{n})$ from $\gamma^{(k-1)}(\underline{n})$ shows that the length of the $o$-blocks between $b$ and $b+s$ must be at least $v_{k} l / u_{k}$. Hence $s-l \geq v_{k} l / u_{k}$ which gives $l \leq u_{k} s / v_{k}$ and $\left(1-\delta_{k-1}\right)(s-l) \geq\left(1-\left(\delta_{k}+u_{k} / v_{k}\right)\right) s$. Thus we can take $\delta_{k}=\delta_{k-1}+u_{k} / v_{k}$ and we have proved (i) since

$$
\delta=\sum_{k=1}^{\infty} \frac{u_{k}}{v_{k}}
$$

Proof of Lemma 2.5 . We first prove that $\left(\underline{n}, \gamma_{j}(\underline{n})\right)$ has the density property for all $\underline{n} \in Q_{j}$ by induction on $j$. This is true for $j=1$ by Lemma 2.3. Assume that $\left(\underline{m}, \gamma_{j-1}(\underline{m})\right)$ has the density property for all $\underline{m} \in Q_{j-1}$. Then clearly $(R(\underline{m}), R \gamma(\underline{m}))$ has the density property for all $\underline{m} \in Q_{j-1}$.

Let $\left\langle a, a^{\prime}\right\rangle$ and $\left\langle a^{\prime}, a^{\prime \prime}\right\rangle$ be two consecutive blocks in $\left(\underline{n}, \gamma_{j}(\underline{n})\right)$. Assume that $n_{a}=1$. By (2.1), $a \in R \gamma\left(\underline{m}_{1}\right)$ and $a^{\prime} \in R \gamma\left(\underline{m}_{2}\right)$ for some $\underline{m}_{1}, \underline{m}_{2} \in Q_{j-1}, R\left(\underline{m}_{1}\right)=$ $R\left(\underline{m}_{2}\right)=\underline{n}$. Let $b$ be the element closest to the right of $a$ in $R \gamma\left(\underline{m}_{1}\right)$, and $b^{\prime}$ the element closest to the left of $a^{\prime}$ in $R \gamma\left(\underline{m}_{2}\right)$. Then $b \geq a^{\prime}$ and $b^{\prime} \leq a$. Since $n_{a}=1$, $\langle a, b\rangle$ is an $o$-block in $\left(\underline{n}, R \gamma\left(\underline{m}_{1}\right)\right)$ and thus $N\left(a, a^{\prime}-1\right)(\underline{n}) \geq 1+(1-\delta)\left(a^{\prime}-1-a\right)$. We see that $\left\langle b^{\prime}, a^{\prime}\right\rangle$ must be an $o$-block and hence $n_{a^{\prime}-1}=1$ and $n_{a^{\prime}}=0$. Consequently $\left\langle a, a^{\prime}\right\rangle$ is an $o$-block and it satisfies (i) and (ii) in Definition 2.1 since $\langle a, b\rangle$ and $\left\langle b^{\prime}, a^{\prime}\right\rangle$ satisfied (i) and (ii). In the same way we show that $n_{a^{\prime}}=0$ implies that $\left\langle a^{\prime}, a^{\prime \prime}\right\rangle$ is an $o$-block and that it satisfies (i) and (ii). Hence $o$ - and $e$-blocks alternate in $\left(\underline{n}, \gamma_{j}(\underline{n})\right)$ and they satisfy (i) and (ii) in Definition 2.1.

Let $A=\left\langle a, a^{\prime}\right\rangle$ be the shortest block in $\left(\underline{n}, \gamma_{j}(\underline{n})\right), a \in R \gamma\left(\underline{m}_{1}\right), R\left(\underline{m}_{1}\right)=\underline{n}$, where $\underline{m}_{1} \in Q_{j-1}$. We take $A$ to be an $o$-block, the other case is similar. If $|A|<u_{j}$ then $\bar{a}^{\prime} \notin R \gamma\left(\underline{m}_{1}\right)$ since all blocks in $\left(R\left(\underline{m}_{1}\right), R \gamma\left(\underline{m}_{1}\right)\right)$ have length $\geq u_{j}$ by definition. To the right of $A$ in $\left(\underline{n}, \gamma_{j}(\underline{n})\right)$ there is an $e$-block $B=\left\langle a^{\prime}, a^{\prime \prime}\right\rangle$ of length $\geq|A|$. By (2.1) the next point to the right of $a$ in $R \gamma\left(\underline{m}_{1}\right)$ is $\geq a^{\prime \prime}$. Hence

$$
N\left(a, a^{\prime \prime}\right) \leq a^{\prime}-a+\delta\left(a^{\prime \prime}-a\right) \leq(1 / 2+\delta)\left(a^{\prime \prime}-a\right) .
$$

But since $\delta \leq 1 / 4$ when $\beta \geq \beta_{0}, 1-\delta>1 / 2+\delta$ and thus this contradicts the density property of $\left(\underline{n}, R \gamma\left(\underline{m}_{1}\right)\right)$ which gives $N\left(a, a^{\prime \prime}\right) \geq(1-\delta)\left(a^{\prime \prime}-a\right)$.

Proof of Lemma 4.1. We prove the first part of lemma, the second part is analogous. The assertion will be proved inductively for $\left(\underline{n}, \gamma^{(k)}(\underline{n})\right), k=0, \ldots, 2 k_{N}$. The case $k=0$ is trivial since $N(x, x+s)(\underline{n}) \leq(1-2 \delta) s$ is impossible. Assume that the assertion is true in $\left(\underline{n}, \gamma^{(k-1)}(\underline{n})\right)$ and let $A=\langle-\infty, a\rangle$ be the first $o$-block in $\left(\underline{n}, \gamma^{(k)}(\underline{n})\right)$. If $\langle-\infty, a\rangle$ is an $o$-block also in $\left(\underline{n}, \gamma^{(k-1)}(\underline{n})\right)$ we are done, otherwise $A=A_{1} B_{1} A_{2} \ldots B_{-1} A_{p}$, where $A_{1}, \ldots, A_{p}$ are $o$-blocks and $B_{1}, \ldots, B_{p-1}$ are $e$ blocks in $\left(\underline{n}, \gamma^{(k-1)}(\underline{n})\right)$. It is clear from the construction that $A_{1}$ and $A_{p}$ both have length $\geq v_{k}$. Let the length of the $e$-blocks which wholly or partly lie in $\langle x, x+s\rangle$ be $y$ and suppose that $j u_{k}<y \leq(j+1) u_{k}$. It follows that $\langle x, x+s\rangle$ contains at least 
$j o$-blocks of length $\geq v_{k}$. Then $s-y \geq j v_{k}$ and $N(x, x+s)(\underline{n}) \geq\left(1-\delta_{k-1}\right)(s-y)$, where $\delta_{k-1}$ is the same as in the proof of Lemma 2.3. We get

$$
\begin{aligned}
N(x, x+s)(\underline{n}) & \geq\left(1-\delta_{k-1}\right)(1-y / s) s \\
& \geq\left(1-\delta_{k-1}\right)\left(1-2 u_{k} / v_{k}\right) s>(1-2 \delta) s,
\end{aligned}
$$

which contradicts our assumption. Hence $y \leq u_{k}$, and from the inequalities $(1-\delta)(s-y) \leq N(x, x+s)(\underline{n}) \leq(1-2 \delta) s$ we find

$$
s \leq \delta^{-1}(1-\delta) y \leq \delta^{-1}(1-\delta) u_{k} .
$$

If $\langle x, x+s\rangle$ lies inside $A_{1}$ the result follows from the induction hypothesis. Otherwise the closest $x$ can be to $-L$ is $L-(s-y) \geq v_{k}-(s-y)$. Thus

$$
\begin{aligned}
x+L & \geq v_{k}-(1-2 \delta) \delta^{-1} u_{k}=2 \delta^{-1} u_{k}^{2}-(1-2 \delta) \delta^{-1} u_{k} \\
& \geq 2 u_{k}^{2} \geq 2\left(\frac{\delta}{1-\delta}\right)^{2} s^{2} .
\end{aligned}
$$

\section{Proof of the Energy Lemma}

In this section we will prove Lemma 3.3. Suppose that $\ldots A^{1} B^{1} A^{2} B^{2} \cdots \rightarrow$ $\ldots A^{1} A^{2} B^{1} B^{2} \ldots$ is the elementary rearrangement that has been performed and that $A^{1}$ is an $o$-block. The case when $A^{1}$ is an $e$-block and thus $B^{2}$ an $o$ block is completely analogous by symmetry. We also assume to begin with that $\alpha<2$. Write $A^{1}=\left\langle a_{1}, b_{1}\right\rangle, B^{1}=\left\langle b_{1}, a_{2}\right\rangle, A^{2}=\left\langle a_{2}, b_{2}\right\rangle$ and $B^{2}=\left\langle b_{2}, a_{3}\right\rangle$ and let $x_{1}=\left|A^{1}\right|, y_{1}=\left|B^{1}\right|, x_{2}=\left|A^{2}\right|, y_{2}=\left|B^{2}\right|$. The elementary rearrangement above is only performed if $x_{1} \geq x_{2}$ and $y_{1} \leq y_{2}$.

Note that we gain energy when two blocks are moved closer to each other and loose energy when they are moved away from each other. If we take all energy losses into consideration but only include the energy gain due to $A^{1}$ and $A^{2}$ having been moved closer to each other, we get the following estimate

$$
\begin{aligned}
H(\underline{n})-H\left(\underline{n}^{\prime}\right) \geq & \sum_{\substack{a_{1} \leq i<b_{1} \\
a_{2} \leq j<b_{2}}} n_{i} n_{j}\left(\frac{1}{\left(j-i-y_{1}\right)^{\alpha}}-\frac{1}{(j-i)^{\alpha}}\right) \\
& -\sum_{\substack{a_{2} \leq i<b_{2} \\
j \geq b_{2}}} n_{i} n_{j}\left(\frac{1}{(j-i)^{\alpha}}-\frac{1}{\left(j-i+y_{1}\right)^{\alpha}}\right)-\sum_{\substack{b_{1} \leq i<a_{2} \\
a_{2} \leq j<b_{2}}} \frac{n_{i} n_{j}}{(j-i)^{\alpha}} \\
& -\sum_{\substack{a_{2} \leq i<b_{2} \\
b_{2} \leq j<a_{3}}} n_{i} n_{j}\left(\frac{1}{(j-i)^{\alpha}}-\frac{1}{\left(j-i+y_{1}\right)^{\alpha}}\right) \\
& -\sum_{\substack{i<b_{1} \\
b_{1} \leq j<a_{2}}} n_{i} n_{j}\left(\frac{1}{(j-i)^{\alpha}}-\frac{1}{\left(j-i+x_{2}\right)^{\alpha}}\right) \\
= & E_{1}-E_{2}-E_{3}-E_{4}-E_{5} .
\end{aligned}
$$

Here $E_{1}$ is the energy gain due to $A^{1}$ and $A^{2}$ moving closer, $E_{2}$ the energy loss due to $A^{2}$ moving away from everything to the right of $B^{2}, E_{3}$ the energy loss due to separating $B^{1}$ and $A^{2}, E_{4}$ the energy loss due to $A^{2}$ moving away from $B^{2}$ and finally $E_{5}$ the energy loss due to $B^{1}$ moving away from $A^{1}$ and everything 
to the left of $A^{1}$. Our problem is to estimate $E_{1}$ to $E_{5}$. Since $n_{i}$ is 0 or 1 we have $n_{i} n_{j} \geq 1-\left(1-n_{i}\right)-\left(1-n_{j}\right)$. If we use this in $E_{1}$ we get an estimate of $E_{1}$ from below by three sums $E_{1}^{(1)}-E_{1}^{(2)}-E_{1}^{(3)}$ corresponding to $1,1-n_{i}$, and $1-n_{j}$ respectively. If we replace all $n_{i}$ and $n_{j}$ in $E_{2}$ by 1 we get an upper bound on $E_{2}$. By rewriting the sums we get the following estimate:

$$
\begin{aligned}
E_{1}^{(1)}-E_{2} \geq & \sum_{i=1}^{x_{1}} \sum_{j=0}^{x_{2}-1}\left(\frac{1}{(j+i)^{\alpha}}-\frac{1}{\left(j+i+y_{1}\right)^{\alpha}}\right) \\
& -\sum_{i=1}^{x_{2}} \sum_{j=0}^{\infty}\left(\frac{1}{\left(j+i+y_{2}\right)^{\alpha}}-\frac{1}{\left(j+i+y_{2}+y_{1}\right)^{\alpha}}\right) \\
\geq & \sum_{i=1}^{x_{2}} \sum_{j=0}^{x_{2}-1}\left(\frac{1}{(j+i)^{\alpha}}-\frac{1}{\left(j+i+y_{1}\right)^{\alpha}}\right) \\
& -\sum_{i=1}^{x_{2}} \sum_{j=0}^{\infty}\left(\frac{1}{\left(j+i+y_{1}\right)^{\alpha}}-\frac{1}{\left(j+i+2 y_{1}\right)^{\alpha}}\right),
\end{aligned}
$$

where in the second inequality we have used $x_{1} \geq x_{2}$ and $y_{1} \leq y_{2}$. Assume first that $y_{1} \geq x_{2}$. The terms in the first double sum in (5.2) are increasing functions of $y_{1}$, whereas the terms in the second double sum are decreasing functions of $y_{1}$. Hence (5.2) is smallest, for fixed $x_{2}$ and $y_{1} \geq x_{2}$, when $y_{1}=x_{2}$. Now assume that $x_{1}>y_{1}$. If we use cancellation between terms in the double sums in (5.2) they can be rewritten as follows:

$$
\begin{aligned}
\sum_{i=1}^{x_{2}} & \left(\sum_{j=0}^{y_{1}-1} \frac{1}{(i+j)^{\alpha}}-\sum_{j=x_{2}}^{x_{2}+y_{1}-1} \frac{1}{(i+j)^{\alpha}}\right)-\sum_{i=1}^{x_{2}} \sum_{j=0}^{y_{1}-1} \frac{1}{\left(i+j+y_{1}\right)^{\alpha}} \\
= & \sum_{i=1}^{x_{2}} \sum_{j=0}^{y_{1}-1}\left(\frac{1}{(i+j)^{\alpha}}-\frac{1}{\left(i+j+x_{2}\right)^{\alpha}}-\frac{1}{\left(i+j+y_{1}\right)^{\alpha}}\right) \\
= & \sum_{j=1}^{y_{1}}\left(\sum_{i=0}^{x_{2}-1} \frac{1}{(i+j)^{\alpha}}-\sum_{i=y_{1}}^{y_{1}+x_{2}-1} \frac{1}{(i+j)^{\alpha}}\right)-\sum_{j=1}^{y_{1}} \sum_{i=0}^{x_{2}-1} \frac{1}{\left(i+j+x_{2}\right)^{\alpha}} \\
= & \sum_{j=1}^{y_{1}}\left(\sum_{i=0}^{y_{1}-1} \frac{1}{(i+j)^{\alpha}}-\sum_{i=x_{2}}^{y_{1}+x_{2}-1} \frac{1}{(i+j)^{\alpha}}\right) \\
& -\sum_{j=1}^{y_{1}} \sum_{i=0}^{\infty}\left(\frac{1}{\left(i+j+x_{2}\right)^{\alpha}}-\frac{1}{\left(i+j+2 x_{2}\right)^{\alpha}}\right) \\
= & \sum_{j=1}^{y_{1}} \sum_{i=0}^{y_{1}-1}\left(\frac{1}{(i+j)^{\alpha}}-\frac{1}{\left(i+j+x_{2}\right)^{\alpha}}\right) \\
& -\sum_{j=1}^{y_{1}} \sum_{i=0}^{\infty}\left(\frac{1}{\left(i+j+x_{2}\right)^{\alpha}}-\frac{1}{\left(i+j+2 x_{2}\right)^{\alpha}}\right) .
\end{aligned}
$$


The same argument as above shows that if $x_{2} \geq y_{1}, y_{1}$ fixed, the last expression in (5.3) is smallest when $x_{2}=y_{1}$. If we put $x=\min \left(x_{2}, y_{1}\right)$ we obtain the estimate

$$
E_{1}^{(1)}-E_{2} \geq \sum_{i=1}^{x} \sum_{j=0}^{x-1}\left(\frac{1}{(i+j)^{\alpha}}-\frac{2}{(i+j+x)^{\alpha}}\right) .
$$

Split this sum into a sum over $i, j$ such that $i+j \leq x$ and a sum where $i+j>x$. If $k=i+j$ we find

$$
\begin{aligned}
E_{1}^{(1)}-E_{2} & \geq \sum_{k=1}^{x} k\left(\frac{1}{k^{\alpha}}-\frac{2}{(k+x)^{\alpha}}\right)+\sum_{k=1}^{x-1} k\left(\frac{1}{(2 x-k)^{\alpha}}-\frac{2}{(3 x-k)^{\alpha}}\right) \\
& =\frac{1}{x^{\alpha}}\left(1-2^{1-\alpha}\right)+\sum_{k=1}^{x-1} \frac{1}{k^{\alpha-1}}\left(1-f\left(\frac{x}{k} ; \alpha\right)\right),
\end{aligned}
$$

where $f(t ; \alpha)=2(1+t)^{-\alpha}+2(3 t-1)^{-\alpha}-(2 t-1)^{-\alpha}$. If $x=1$ the sum in (5.5) is zero. At the end of this section we will show that

$$
\sup _{t \geq 1} f(t ; \alpha)=1-d_{\alpha}<1
$$

Define $g(x)=1+(2-\alpha)^{-1}\left(x^{2-\alpha}-1\right)$. If we use (5.6) in (5.5) we get

$$
E_{1}^{(1)}-E_{2} \geq \min \left\{1-2^{1-\alpha}, d_{\alpha}\right\} \sum_{k=1}^{x-1} \frac{1}{k^{\alpha-1}} \geq d_{\alpha}^{\prime} g(x) .
$$

We will now show how $E_{5}$ and $E_{1}^{(2)}$ are estimated. $E_{3}, E_{4}$ and $E_{1}^{(3)}$ are estimated similarly. By the density property

$$
\sum_{j=b_{1}}^{b_{1}+k} n_{j} \leq \delta k, \quad 0 \leq k \leq y_{1}-1 .
$$

If we use $n_{i} \leq 1$, summation by parts and estimation of sums by integrals we get

$$
\begin{aligned}
E_{5} & \leq \sum_{i<b_{1}} \sum_{j=b_{1}}^{a_{2}-1} n_{j}\left(\frac{1}{(j+i)^{\alpha}}-\frac{1}{\left(j+i+x_{2}\right)^{\alpha}}\right) \\
& \leq \delta \sum_{i=1}^{\infty} \sum_{j=1}^{y_{1}-1}\left(\frac{1}{(i+j)^{\alpha}}-\frac{1}{\left(i+j+x_{2}\right)^{\alpha}}\right) \\
& \leq \delta \sum_{i=1}^{x_{2}} \sum_{j=1}^{y_{1}} \frac{1}{(i+j)^{\alpha}} \leq \frac{\delta}{\alpha-1} g(x),
\end{aligned}
$$

where $x=\min \left(x_{2}, y_{1}\right)$. The density property gives

$$
\sum_{i=1}^{k} 1-n_{b_{1}-1} \leq \delta(k-1), \quad k=1, \ldots, x_{1} .
$$


Using this estimate in a summation by parts we find

$$
\begin{aligned}
E_{1}^{(2)} & =\sum_{i=a_{1}}^{b_{1}-1} \sum_{j=a_{2}}^{b_{2}-1}\left(1-n_{i}\right)\left(\frac{1}{\left(j-i+y_{1}\right)^{\alpha}}-\frac{1}{(j-i)^{\alpha}}\right) \\
& =\sum_{i=1}^{x_{1}} \sum_{j=0}^{x_{2}-1}\left(1-n_{b_{1}-i}\right)\left(\frac{1}{(i+j)^{\alpha}}-\frac{1}{\left(i+j+y_{1}\right)^{\alpha}}\right) \\
& =\delta \sum_{i=2}^{\infty} \sum_{j=0}^{x_{2}-1}\left(\frac{1}{(i+j)^{\alpha}}-\frac{1}{\left(i+j+y_{1}\right)^{\alpha}}\right) \\
& =\delta \sum_{i=1}^{y_{1}} \sum_{j=1}^{x_{2}} \frac{1}{(i+j)^{\alpha}}=\frac{\delta}{\alpha-1} g(x) .
\end{aligned}
$$

A similar argument gives the same estimate for $E_{1}^{(3)}, E_{3}$ and $E_{4}$, and together with (5.7) this gives

$$
H(\underline{n})-H\left(\underline{n}^{\prime}\right) \geq\left(d_{\alpha}^{\prime}-\frac{5 \delta}{\alpha-1}\right) .
$$

Note that $g(x) \geq 1+\log x$ and that $g(x) \rightarrow 1+\log x$ as $\alpha \rightarrow 2-$. Put $d_{\alpha}^{\prime}=$ $4 \kappa / \log 2$ and assume that $\delta \leq \frac{1}{5}(\alpha-1) 2 \kappa / \log 2$. Since $x \geq 2^{j-1}$, (5.8) gives that $H(\underline{n})-H\left(\underline{n}^{\prime}\right) \geq 2 \kappa j$ which is what we wanted to prove.

We have proved Lemma 3.3 except for (5.6), the proof of which we outline below. We have

$$
\frac{\partial f}{\partial \alpha}=-2 \frac{\log (1+t)}{(t+1)^{\alpha}}-2 \frac{\log (3 t-1)}{(3 t-1)^{\alpha}}+\frac{\log (2 t-1)}{(2 t-1)^{\alpha}} .
$$

With some work the right inequality in

$$
\frac{\log (2 t-1)}{(2 t-1)^{\alpha}} \leq 2 \frac{\log (1+t)}{(1+t)^{\alpha}} \Leftrightarrow \frac{\log (2 t-1)}{\log (1+t)} \leq 2\left(\frac{2 t-1}{t+1}\right)^{\alpha}
$$

can be poved for $t \geq 1$ and consequently $\partial f / \partial \alpha<0$ if $t \geq 1$. When $t>1$ it is not difficult to see that $f(t ; \alpha)<1$. When $1<\alpha \leq 2, f(1 ; \alpha) \geq 0$ and $(\partial f / \partial \alpha)(1 ; \alpha)>0$. Hence $f(x ; \alpha)$, for fixed $\alpha$, attains its maximum at some point $t_{\alpha}>1 ; t_{\alpha}$ only depends on $\alpha$. Thus

$$
f(t ; \alpha) \leq f\left(t_{\alpha} ; \alpha\right)<f\left(t_{\alpha} ; 1\right)<1
$$

for all $t \geq 1$. If we put $1-d_{\alpha}=f\left(t_{\alpha} ; 1\right)$ we are done.

\section{Proof of Theorem 1.3}

Fix a $\beta$ such that the free energy $f(\varrho, \beta)$ defined by (1.7) is a strictly convex function of $\varrho$ and fix $N, L$. Let $\underline{n} \in Q$ be a configuration and let $x_{\mu}(\underline{n})$ denote the $\mu:^{\text {th }}$ occupied position in $\Lambda$, i.e. the position of the $\mu:{ }^{\text {th }}$ particle, $\mu=1, \ldots, N$. For a given small $\varepsilon>0$ we define

$$
I_{\mu}=\{x \in \Lambda ;|x / L-\mu / N|>\varepsilon\}
$$

and

$$
M_{\varepsilon, \mu}=\left\{\underline{n} \in Q ; x_{\mu}(\underline{n}) \in I_{\mu}\right\} .
$$


Let $v=v(N, L)$ be such that

$$
\sum_{\underline{n} \in M_{\varepsilon, \mu}} e^{-\beta H(\underline{n})}
$$

is maximal when $\mu=v$ and let $\xi=\xi(N, L)$ be such that

$$
\sum_{\underline{n} \in M_{\varepsilon, \mu} ; x_{v}(\underline{n})=x} e^{-\beta H(\underline{n})}
$$

is maximal when $x=\xi$. Note that $\xi \in I_{v}$. If we define

$$
H_{1}(\underline{n})=-\sum_{\substack{i, j \in[1, \xi) \cap \Lambda \\ i \neq j}} \frac{n_{i} n_{j}}{|i-j|^{\alpha}}, \quad H_{2}(\underline{n})=-\sum_{\substack{i, j \in(\xi, L) \cap \Lambda \\ i \neq j}} \frac{n_{i} n_{j}}{|i-j|^{\alpha}},
$$

then $H(\underline{n})-H_{1}(\underline{n})-H_{2}(\underline{n}) \geq o(N)$, where $o(N)$ is positive and does not depend on $\underline{n}$. Let $M_{\varepsilon}=\bigcup_{\mu=1}^{N} M_{\varepsilon, \mu}$. Then

$$
\begin{aligned}
\sum_{\underline{n} \in M_{\varepsilon}} e^{-\beta H(\underline{n})} & \leq \sum_{\mu=1}^{N} \sum_{\underline{n} \in M_{\varepsilon, \mu}} e^{-\beta H(\underline{n})} \\
& \leq N \sum_{x \in I_{v}}\left(\sum_{\underline{\underline{n} \in M_{\varepsilon, \mu} ; x_{v}(\underline{n})=x}} e^{-\beta H(\underline{n})}\right) \\
& \leq N L \sum_{\underline{\underline{n}} \in M_{\varepsilon, \mu} ; x_{v}(\underline{n})=\xi} e^{-\beta H(\underline{n})} \leq N L \sum_{\underline{n} ; x_{v}(\underline{n})=\xi} e^{\beta H(\underline{n})} \\
& \leq e^{o(N)} Z(v-1, \xi-1) Z(N-v, L-\xi) .
\end{aligned}
$$

We want to show that

$$
\limsup _{\Lambda \rightarrow Z^{+}} P\left(M_{\varepsilon}\right)^{1 / L} \leq c<1,
$$

where $c$ is a constant (that depends on $\varepsilon$ ). Choose a subsequence $N_{p} \rightarrow \infty$, $L_{p} \rightarrow \infty$ and $N_{p} / L_{p} \rightarrow \varrho$ as $p \rightarrow \infty$ which gives the upper limit in (6.2) and such that

$$
t=\lim _{p \rightarrow \infty} \frac{v\left(N_{p}, L_{p}\right)}{N_{p}} \quad \text { and } s=\lim _{p \rightarrow \infty} \frac{\xi\left(N_{p}, L_{p}\right)}{L_{p}}
$$

exist. Now, using (5.1) and the definition of free energy we see that

$$
\limsup _{\Lambda \rightarrow Z^{+}} P\left(M_{\varepsilon}\right)^{1 / L} \leq \exp \beta\left[f(\varrho, \beta)-s f\left(\frac{t}{s} \varrho, \beta\right)-(1-s) f\left(\frac{1-t}{1-s} \varrho, \beta\right)\right] .
$$

Observe that $\xi \in I_{v}$ implies $|\xi / L-v / N| \geq \varepsilon$ and consequently $|s-t| \geq \varepsilon$. Since $\xi \geq v$ and $N-v \leq L-\xi$ we must have $0<s<1$. Thus $\varrho t / s \neq \varrho(1-t) /(1-s)$ and the strict convexity of $f$ gives

$$
f(\varrho, \beta)-s f\left(\frac{t}{s} \varrho, \beta\right)-(1-s) f\left(\frac{1-t}{1-s} \varrho, \beta\right)<0,
$$

and we have proved (6.2). It follows from (6.2) that

$$
\limsup _{\Lambda \rightarrow Z^{+}} P\left(M_{\varepsilon}\right)=0
$$


for every fixed $\varepsilon>0$. We will prove that

$$
Q \backslash M_{\varepsilon^{\prime}} \subset\left\{\underline{n} \in Q ; \varrho-\varepsilon \leq d\left(\tau_{1}, \tau_{2}\right)(\underline{n}) \leq \varrho+\varepsilon\right\},
$$

where $\varepsilon^{\prime}=\varepsilon / 3 \varrho$. Equations (6.3) and (6.4) together prove Theorem 1.3.

If $\underline{n} \in Q \backslash M_{\varepsilon^{\prime}}$, then $\left|x_{\mu}(\underline{n}) / L-\mu / N\right| \leq \varepsilon^{\prime}$ for $\mu=1, \ldots, N$. Thus for each $\tau, 0<\tau<1$, we have $\mu>\left(\tau+\varepsilon^{\prime}\right) N$, which gives $x_{\mu}(\underline{n})>\tau L$, and hence $N(0, \tau L)(\underline{n}) \leq \mu-1$. Consequently $N(0, \tau L) \leq\left(\tau+\varepsilon^{\prime}\right) N$. Similarly $\mu \leq\left(\tau-\varepsilon^{\prime}\right) N$ implies $N(0, \tau L)(\underline{n}) \geq\left[\left(\tau-\varepsilon^{\prime}\right) N\right]$. These two estimates of $N(0, \tau L)(\underline{n})$ give

$$
\varrho\left(1-3 \varepsilon^{\prime}\right) \leq d\left(\tau_{1}, \tau_{2}\right)(\underline{n}) \leq \varrho\left(1+3 \varepsilon^{\prime}\right),
$$

and we have proved (6.4).

\section{References}

1. Aizenman, M., Chayes, J.T., Chayes, L., Newman, C.M.: J. Stat. Phys. 50, 1 (1988)

2. Dyson, F.J.: Commun. Math. Phys. 12, 212 (1969) and 21, 269 (1971)

3. Ellis, R.S.: Entropy, large deviations and statistical mechanics. Berlin, Heidelberg, New York: Springer 1985

4. Fröhlich, J., Spencer, T.: Commun. Math. Phys. 84, 87-101 (1982)

5. Johansson, K.: Separation of phases at low temperatures in a one-dimensional continuous gas. Commun. Math. Phys. 141, 259-278 (1991)

6. Landau, L.D., Lifshitz, E.M.: Statistical physics. London: Pergamon Press 1958

7. Rockafellar, R.T.: Convex analysis. Princeton, NJ: Princeton University Press 1970

8. Ruelle, D.: Statistical mechanics. New York: Benjamin 1969

9. Thouless, D.J.: Phys. Rev. 187, 732-733 (1969)

Communicated by M. Aizenman 
